Bull. Chem. Soc. Ethiop. 2017, 31(2), 337-344.

ISSN 1011-3924

(c) 2017 Chemical Society of Ethiopia and The Authors

Printed in Ethiopia

DOI: http://dx.doi.org/10.4314/bcse.v31i2.15

\title{
SYNTHESIS AND IN-VITRO STUDIES OF SOME NEW QUINOLINE 1,3,4- THIADIAZOLO PYRIMIDIN DERIVATIVES
}

\author{
Kishore Kumar Valluri ${ }^{1}$, I.V. Kasi Viswanath ${ }^{1 *}$, P.V.V.S. Naga Raju ${ }^{1}$, B. Rajasekher ${ }^{2}$ and \\ B. Rama Dasu ${ }^{1}$ \\ ${ }^{1}$ Department of Chemistry, K.L. University, Green Fields, Vaddeswram, Guntur, A.P, India \\ ${ }^{2}$ Vasudha Pharmaceuticals, Visakhapatnam, India
}

(Received April 7, 2016; revised August 22, 2017)

\begin{abstract}
A series of eight new quinoline associated 1,3,4-thiadiazolo pyrimidin derivatives (5a-h) have been developed using 4-amino-8-fluoro-quinoline-3-carboxylic acid ethyl ester (1) as raw material and by involving 8-fluoro-4-methylsulfanylthiocarbonylamino-quinoline-3-carboxylic acid ethyl ester (2), 8-fluoro-4hydrazine thiocarbonylamino-quinoline-3-carboxylic acid ethyl ester (3) and 3-amino-7-fluoro-2-mercapto-3 $\mathrm{H}$ pyrimido-[5,4-c] quinolin-4-one (4) as intermediates. The title compounds after structure elucidation were used in vitro to find their antibacterial ability towards different micro-organisms.
\end{abstract}

KEY WORDS: Synthesis, Quinoline, 1,3,4-thiadiazolo pyrimidin, Spectral data, Potential activity

\section{INTRODUCTION}

The quinoline skeleton is often used for the design of many synthetic compounds with diverse pharmacological properties such as anti-inflammatory [1], antimicrobial [2], cytotoxic [3], antitumor [4], antimalarial [5], anti-cancer [6], HIV protease inhibitor [7] and anti-viral [8] activities. It is known that many 1,3,4-thiadiazoles have biological activities like antibacterial [9], antimycobacterial [10], antimycotic [11], antifungal [12], antidepression [13], analgesic [14] and anti-inflammatory [15]. Nitrogen containing heterocyclic ring such as pyrimidine and its derivatives have been reported as anti-microbial [16], analgesic, anti-viral, anti-inflammatory [17], anti-HIV [18], anti-tubercular [19], anti-tumour [20], anti-neoplastic [21], anti-malarial [22], diuretic [23], cardiovascular [24] agents.

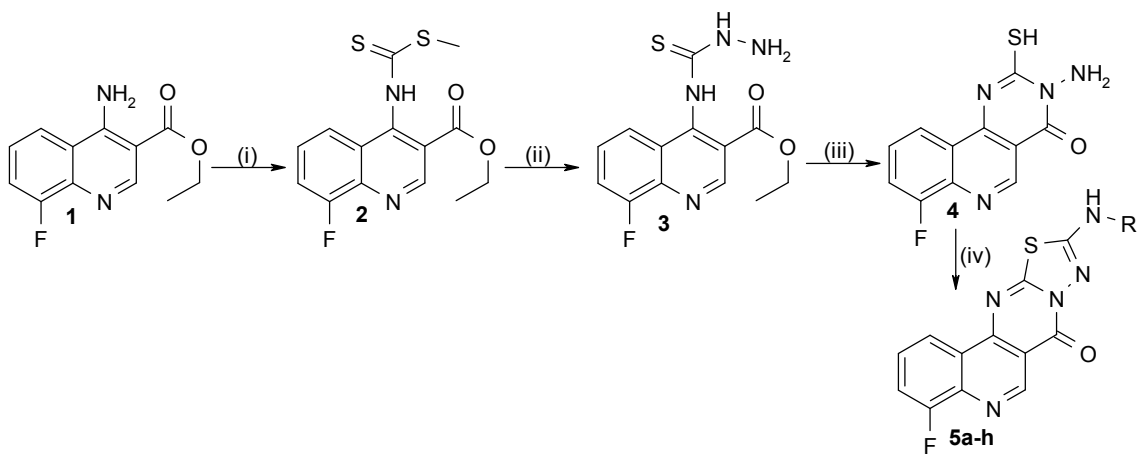

Scheme 1. (i) $\mathrm{CS}_{2}, \mathrm{NaOH}, \mathrm{DMSO}, \mathrm{RT}, 2 \mathrm{~h}$; (ii) $\mathrm{NH}_{2}-\mathrm{NH}_{2}$, EtOH, reflux, 6 h; (iii) EtOH, reflux, $4 \mathrm{~h}$; (iv) ArNCS, $\mathrm{K}_{2} \mathrm{CO}_{3}$, DMF, reflux, 20-22 h 5 (a-h): $\mathrm{R}=$ a) $\mathrm{C}_{6} \mathrm{H}_{5}$; b) $4-\mathrm{CH}_{3}-\mathrm{C}_{6} \mathrm{H}_{4}$; c) $3-\mathrm{OCH}_{3}-\mathrm{C}_{6} \mathrm{H}_{4}$; d) $4-\mathrm{OCH}_{3}-\mathrm{C}_{6} \mathrm{H}_{4}$; e) $3-\mathrm{Cl}-\mathrm{C}_{6} \mathrm{H}_{4}$; f) $4-\mathrm{Cl}-\mathrm{C}_{6} \mathrm{H}_{4}$; g) $3 \mathrm{NO}_{2}-\mathrm{C}_{6} \mathrm{H}_{4}$; h) $4-\mathrm{NO}_{2}-\mathrm{C}_{6} \mathrm{H}_{4}$.

*Corresponding author. E-mail: viswanath_fed@kluniversity.in

This work is licensed under the Creative Commons Attribution 4.0 International License 
Here in, we report a series of eight new quinoline associated 1,3,4-thiadiazolo pyrimidin derivatives. Compound 5e displayed activity more than standard benzyl pencillin against $B$. subtilis and considerable activity against streptomycin, and compounds $\mathbf{5 b}$ and $\mathbf{5 f}$ are significant active towards M. luteus compared to standard drug. The general synthetic scheme is presented in Scheme 1.

\section{EXPERIMENTAL}

All the reagents and solvents were used as purchased without further purification. Melting points were determined on a Fisher-Johns melting point apparatus and are uncorrected. IR spectra were obtained on a Perkin-Elmer BX serried FTIR 5000 spectrometer using $\mathrm{KBr}$ pellet. NMR spectra were recorded on a Varian $300 \mathrm{MHz}$ spectrometer for ${ }^{1} \mathrm{H}-\mathrm{NMR}$ and $100 \mathrm{MHz}$ for ${ }^{13} \mathrm{C}-\mathrm{NMR}$. The chemical shifts were reported as ppm down field using TMS as an internal standard. Mass spectra were recorded on a VG-Micromass $7070 \mathrm{H}$ spectrometer operating at 70 $\mathrm{eV}$.

The antimicrobial studies were conducted by broth method. The broth culture is incubated in non- $\mathrm{CO}_{2}$ incubator at $35^{\circ} \mathrm{C}$ until it achieves or exceeds the turbidity of the $0.5 \mathrm{McF}$ arland standard (usually 2 to 6 hours). By obtaining isolated colonies of bacterial strains to test and combine the colonies and culture in rich media, incubate overnight and count colonies. The turbidity of the actively growing broth culture is adjusted with sterile saline or broth to obtain a turbidity optically comparable to that of the $0.5 \mathrm{McF}$ arland standard and verified $\mathrm{cfu} / \mathrm{mL}$ count of visible media and the collective results were noted.

Synthesis of 8-fluoro-4-methylsulfanylthiocarbonylamino-quinoline-3-carboxylic acid ethyl ester (2)

To a mixture of 4-amino-8-fluoro-quinoline-3-carboxylic acid ethyl ester (1) $(0.01 \mathrm{~mol})$ in dimethyl sulphoxide $(10 \mathrm{~mL})$ at room temperature was added carbon disulfide $(1.4 \mathrm{~mL})$ and aqueous sodium hydroxide $(1.0 \mathrm{~mL})$ solution in drop wise. After $30 \mathrm{~min}$ dimethyl sulfate $(2.3 \mathrm{~g})$ was added under cold conditions by keeping in an ice bath. The reaction mixture then constantly stirred at room temperature for $1.5 \mathrm{~h}$. After completion of the reaction (monitored by TLC), the resultant solution was poured in ice water. The solid that separated out was filtered, dried and recrystallized from ethanol to get pure 8-fluoro-4-methylsulfanylthiocarbonylamino-quinoline3-carboxylic acid ethyl ester (2).

Synthesis of 8-fluoro-4-hydrazine thiocarbonylamino-quinoline-3-carboxylic acid ethyl ester (3)

To the mixture of 8-fluoro-4-methylsulfanylthiocarbonylamino-quinoline-3-carboxylic acid ethyl ester (2) $(0.01 \mathrm{~mol})$ in ethanol $(10 \mathrm{~mL})$ was added hydrazine hydrate $(15 \mathrm{~mL})$ and the resulted solution was refluxed for $6 \mathrm{~h}$ on uniform stirring. After completion of the reaction (examined by TLC), the mixture was cooled to room temperature, poured in ice-cold water (15 $\mathrm{mL}$ ) and filtered. The obtained crude solid was recrystallized from ethanol to give 8-fluoro-4hydrazine thiocarbonylamino-quinoline-3-carboxylic acid ethyl ester (3).

Synthesis of 3-amino-7-fluoro-2-mercapto-3H-pyrimido-[5,4-c]quinolin-4-one (4)

A mixture of 8-fluoro-4-hydrazine thiocarbonylamino-quinoline-3-carboxylic acid ethyl ester (3) $(0.01 \mathrm{~mol})$ in ethanol $(10 \mathrm{~mL})$ was heated under reflux with steady stirring for $4 \mathrm{~h}$. After completion of the reaction (watched by TLC), the residual mass was poured over crushed ice and the precipitated crude product was filtered, washed with water, dried and recrystallized from ethanol to get the pure 3-amino-7-fluoro-2-mercapto-3H-pyrimido-[5,4-c]quinolin-4-one (4). 
Synthesis of 2-arylamino-8-fluoro-[1,3,4] thiadiazolo-[3,2-a]pyrimidin-[5,4-c]quinolin-5-ones $(5 a-h)$

A mixture of 3-amino-7-fluoro-2-mercapto-3H-pyrimido-[5,4-c]quinolin-4-one (4) $(0.01 \mathrm{~mol})$, small amount of anhydrous potassium carbonate and suitable aryl isothiocyantes in DMF (15 $\mathrm{mL}$ ) was refluxed with consistent stirring for 20-22 h. After completion of the reaction (monitored by TLC), the reaction mixture was cooled to room temperature and poured in crushed ice. The resulted solid was filtered off. The crude product thus obtained was purified by column chromatography on silica gel with hexane-ethyl acetate as eluent to afford corresponding 2-arylamino-8-fluoro-[1,3,4] thiadiazolo-[3,2-a]pyrimidin-[5,4-c]quinolin-5-ones $(\mathbf{5 a}-\mathbf{h})$ in pure form.

\section{Physical and spectral data}

8-Fluoro-4-methylsulfanylthiocarbonylamino-quinoline-3-carboxylic acid ethyl ester (2). Yellow solid, yield: 70\%, mp: 184-186 ${ }^{\circ} \mathrm{C}$, IR (KBr): 3347 (N-H), 3052 (C-H, Ar), 2960 (C-H, $\left.\mathrm{CH}_{3}\right), 1740(\mathrm{C}=\mathrm{O}), 1640(\mathrm{C}=\mathrm{C}, \mathrm{Ar}), 1576(\mathrm{C}=\mathrm{N}), 1240(\mathrm{C}=\mathrm{S}), 1085(\mathrm{C}-\mathrm{O}) \mathrm{cm}^{-1} ;{ }^{1} \mathrm{H}$ NMR $(300$ MHz, DMSO-d $\left.{ }_{6}\right): \delta$ ppm $12.21(\mathrm{~s}, 1 \mathrm{H}, \mathrm{NH}), 7.84(\mathrm{~s}, 1 \mathrm{H}, \mathrm{Ar}-\mathrm{H}), 7.68-7.42(\mathrm{~m}, 3 \mathrm{H}, \mathrm{Ar}-\mathrm{H}), 3.68$ $\left(\mathrm{q}, 2 \mathrm{H}, \mathrm{J}=5.4 \mathrm{~Hz}, \mathrm{OCH}_{2}\right), 2.89\left(\mathrm{~s}, 3 \mathrm{H}, \mathrm{SCH}_{3}\right), 1.82\left(\mathrm{t}, 3 \mathrm{H}, \mathrm{J}=5.4 \mathrm{~Hz}, \mathrm{CH}_{3}\right) .{ }^{13} \mathrm{C} \mathrm{NMR}(100$ MHz, DMSO-d $\left.{ }_{6}\right): \delta$ ppm 178.9, 165.2, 158.7, 155.4, 152.3, 139.5, 124.2, 118.7, 116.3, 113.8, 106.9, 54.7, 14.8, 12.8. MS: $324 \mathrm{~m} / \mathrm{z}\left(\mathrm{M}^{+}\right)$. Elemental analysis: calculated for $\mathrm{C}_{14} \mathrm{H}_{13} \mathrm{FN}_{2} \mathrm{O}_{2} \mathrm{~S}_{2}$ : C-51.84, H-4.04, F-5.86, N-8.64, O-9.86, S-19.77. Found: C-49.98, H-3.87, F-5.12, N-8.28, O9.02, S-18.84.

8-Fluoro-4-hydrazine thiocarbonylamino-quinoline-3-carboxylic acid ethyl ester (3). Brown solid, yield: $69 \%$, mp: $169-171{ }^{\circ} \mathrm{C}$, IR $(\mathrm{KBr}): 3358(\mathrm{~N}-\mathrm{H}), 3245(\mathrm{~N}-\mathrm{H}), 3048(\mathrm{C}-\mathrm{H}, \mathrm{Ar}), 2954$ $\left(\mathrm{C}-\mathrm{H}, \mathrm{CH}_{3}\right), 1738(\mathrm{C}=\mathrm{O}), 1648(\mathrm{C}=\mathrm{C}, \mathrm{Ar}), 1580(\mathrm{C}=\mathrm{N}), 1236(\mathrm{C}=\mathrm{S}), 1092(\mathrm{C}-\mathrm{O}) \mathrm{cm}^{-1} ;{ }^{1} \mathrm{H}$ NMR (300 MHz, DMSO-d $)_{6}$ ) $\delta$ ppm $12.36(\mathrm{~s}, 1 \mathrm{H}, \mathrm{NH}), 7.89$ (s, 1H, Ar-H), $7.68(\mathrm{~s}, 1 \mathrm{H}, \mathrm{NH}), 7.60-$ $7.47(\mathrm{~m}, 3 \mathrm{H}, \mathrm{Ar}-\mathrm{H}), 4.42\left(\mathrm{~s}, 2 \mathrm{H}, \mathrm{NH}_{2}\right), 3.61\left(\mathrm{q}, 2 \mathrm{H}, \mathrm{J}=5.0 \mathrm{~Hz}, \mathrm{OCH}_{2}\right), 1.79$ (t, 3H, J = 5.0 Hz, $\left.\mathrm{CH}_{3}\right) .{ }^{13} \mathrm{C}$ NMR (100 MHz, DMSO-d 6 ): $\delta$ ppm 174.3, 165.3, 156.7, 150.2, 147.6, 143.2, 124.8, 120.3, 118.7, 113.5, 105.8, 57.4, 14.2. MS: $308 \mathrm{~m} / \mathrm{z}\left(\mathrm{M}^{+}\right)$. Elemental analysis: calculated for $\mathrm{C}_{13} \mathrm{H}_{13} \mathrm{FN}_{4} \mathrm{O}_{2} \mathrm{~S}$ : C-50.64, H-4.25, F-6.16, N-17.17, O-10.38, S-10.40. Found: C-49.16, H-4.12, F-6.10, N-17.00, O-9.84, S-9.85.

3-Amino-7-fluoro-2-mercapto-3H-pyrimido-[5,4-c]quinolin-4-one (4). Pale yellow solid, yield: 71\%, mp: 154-156 ${ }^{\circ} \mathrm{C}$, IR (KBr): $3214(\mathrm{~N}-\mathrm{H}), 3056(\mathrm{C}-\mathrm{H}, \mathrm{Ar}), 2966\left(\mathrm{C}-\mathrm{H}, \mathrm{CH}_{3}\right), 2562(\mathrm{~S}-\mathrm{H})$, $1684(\mathrm{C}=\mathrm{O}), 1656(\mathrm{C}=\mathrm{C}, \mathrm{Ar}), 1588(\mathrm{C}=\mathrm{N}) \mathrm{cm}^{-1} ;{ }^{1} \mathrm{H}$ NMR $\left(300 \mathrm{MHz}, \mathrm{DMSO}-\mathrm{d}_{6}\right): \delta \mathrm{ppm} 7.84(\mathrm{~s}$, 1H, Ar-H), 7.63-7.58 (m, 3H Ar-H), 5.79 (s, 2H, NH $), 3.21$ (s, 1H, S-H). ${ }^{13} \mathrm{C}$ NMR (100 MHz, DMSO-d $\left.{ }_{6}\right): \delta$ ppm 165.7, 163.2, 158.6, 157.4, 147.8, 136.5, 127.8, 123.5, 122.8, 122.0, 113.5. MS: $262 \mathrm{~m} / \mathrm{z}\left(\mathrm{M}^{+}\right)$. Elemental analysis: calculated for $\mathrm{C}_{11} \mathrm{H}_{7} \mathrm{FN}_{4} \mathrm{OS}$ : C-50.38, H-2.69, F-7.24, N21.36, O-6.10, S-12.23. Found: C-49.68, H-2.45, F-7.12, N-20.87, O-5.89, S-11.98.

2-Phentlamino-8-fluoro-[1,3,4] thiadiazolo-[3,2-a]pyrimidin-[5,4-c]quinolin-5-one (5a). Yellow solid, yield: 70\%, mp: 144-146 ${ }^{\circ} \mathrm{C}$, IR (KBr): $3164\left(\mathrm{~N}-\mathrm{H}, \mathrm{NH}_{2}\right), 3062(\mathrm{C}-\mathrm{H}, \mathrm{Ar}), 1683(\mathrm{C}=\mathrm{O})$, $1615(\mathrm{C}=\mathrm{C}, \mathrm{Ar}), 1577(\mathrm{C}=\mathrm{N}) \mathrm{cm}^{-1} ;{ }^{1} \mathrm{H}$ NMR $\left(300 \mathrm{MHz}, \mathrm{DMSO}-\mathrm{d}_{6}\right): \delta \mathrm{ppm} 9.69(\mathrm{~s}, 1 \mathrm{H}, \mathrm{NH})$, $7.89(\mathrm{~s}, 1 \mathrm{H}, \mathrm{Ar}-\mathrm{H}), 7.74-7.48(\mathrm{~m}, 8 \mathrm{H}, \mathrm{Ar}-\mathrm{H}) .{ }^{13} \mathrm{C}$ NMR (100 MHz, DMSO-d $\left.{ }_{6}\right): \delta$ ppm 168.9, 162.3, 160.2, 156.7, 152.3, 147.2, 144.2, 136.4, 127.6, 125.7, 123.6, 122.7, 121.4, 119.5, 116.8, 114.2. MS: $363 \mathrm{~m} / \mathrm{z}\left(\mathrm{M}^{+}\right)$. Elemental analysis: calculated for $\mathrm{C}_{18} \mathrm{H}_{10} \mathrm{FN}_{5} \mathrm{OS}$ : C-59.50, H-2.77, F5.23, N-19.27, O-4.40, S-8.82. Found: C-58.02, H-2.61, F-5.02, N-18.47, O-4.21, S-8.26. 
2-(4-Methyl phentlamino)-8-fluoro-[1,3,4] thiadiazolo-[3,2-a]pyrimidin-[5,4-c]quinolin-5-one (5b). Pale brown solid, yield: 68\%, mp: $135-137{ }^{\circ} \mathrm{C}$, IR $(\mathrm{KBr}): 3178\left(\mathrm{~N}-\mathrm{H}, \mathrm{NH}_{2}\right), 3068(\mathrm{C}-\mathrm{H}$, Ar), $1679(\mathrm{C}=\mathrm{O}), 1628(\mathrm{C}=\mathrm{C}, \mathrm{Ar}), 1584(\mathrm{C}=\mathrm{N}) \mathrm{cm}^{-1} ;{ }^{1} \mathrm{H}$ NMR $\left(300 \mathrm{MHz}, \mathrm{DMSO}-\mathrm{d}_{6}\right): \delta \mathrm{ppm}$ $9.62(\mathrm{~s}, 1 \mathrm{H}, \mathrm{NH}), 7.69(\mathrm{~s}, 1 \mathrm{H}, \mathrm{Ar}-\mathrm{H}), 7.65-7.51(\mathrm{~m}, 3 \mathrm{H}, \mathrm{Ar}-\mathrm{H}), 7.64(\mathrm{~d}, 2 \mathrm{H}, \mathrm{J}=7.4 \mathrm{~Hz}, \mathrm{Ar}-\mathrm{H})$, $7.42(\mathrm{~d}, 2 \mathrm{H}, \mathrm{J}=7.4 \mathrm{~Hz}, \mathrm{Ar}-\mathrm{H}), 2.15\left(\mathrm{~s}, 3 \mathrm{H}, \mathrm{CH}_{3}\right) .{ }^{13} \mathrm{C}$ NMR $\left.(100 \mathrm{MHz}, \text { DMSO-d })_{6}\right): \delta \mathrm{ppm}$ 166.7, 161.8, 158.7, 155.9, 154.2, 148.6, 143.2, 137.4, 129.6, 124.7, 122.0, 121.8, 120.3, 118.4, 115.6, 113.8, 36.3. MS: $377 \mathrm{~m} / \mathrm{z}\left(\mathrm{M}^{+}\right)$. Elemental analysis: calculated for $\mathrm{C}_{19} \mathrm{H}_{12} \mathrm{FN}_{5} \mathrm{OS}$ : C60.47, H-3.20, F-5.03, N-18.56, O-4.24, S-8.50. Found: C-58.98, H-3.12, F-4.87, N-17.98, O4.12, S-8.14.

2-(3-Methoxy phentlamino)-8-fluoro-[1,3,4] thiadiazolo-[3,2-a]pyrimidin-[5,4-c]quinolin-5-one (5c). White solid, yield: $69 \%$, mp: $128-130{ }^{\circ} \mathrm{C}$, IR $(\mathrm{KBr}): 3162\left(\mathrm{~N}-\mathrm{H}, \mathrm{NH}_{2}\right), 3073(\mathrm{C}-\mathrm{H}, \mathrm{Ar})$, $1684(\mathrm{C}=\mathrm{O}), 1644(\mathrm{C}=\mathrm{C}, \mathrm{Ar}), 1578(\mathrm{C}=\mathrm{N}), 1158(\mathrm{C}-\mathrm{O}) \mathrm{cm}^{-1} ;{ }^{1} \mathrm{H}$ NMR $\left(300 \mathrm{MHz}, \mathrm{DMSO}-\mathrm{d}_{6}\right): \delta$ ppm 9.55 (s, 1H, NH), 7.65 (s, 1H, Ar-H), 7.72-7.55 (m, 6H, Ar-H), 7.64 (s, 1H, Ar-H), 3.21 (s, $\left.3 \mathrm{H}, \mathrm{OCH}_{3}\right) .{ }^{13} \mathrm{C}$ NMR $(100 \mathrm{MHz}$, DMSO-d 6 ): $\delta$ ppm 166.7, 164.0, 163.6, 155.8, 154.7, 149.5, $146.1,137.4,129.6,128.4,127.5,126.3,125.3,124.7,122.8,121.7,119.5,116.3,44.8$. MS: $393 \mathrm{~m} / \mathrm{z}\left(\mathrm{M}^{+}\right)$. Elemental analysis: calculated for $\mathrm{C}_{19} \mathrm{H}_{12} \mathrm{FN}_{5} \mathrm{O}_{2} \mathrm{~S}$ : C-58.01, H-3.07, F-4.83, N17.80, O-8.13, S-5.15. Found: C-56.23, H-2.98, F-4.54, N-17.02, O-7.84, S-5.02.

2-(4-Methoxy phentlamino)-8-fluoro-[1,3,4] thiadiazolo-[3,2-a]pyrimidin-[5,4-c]quinolin-5-one (5d). Yellow solid, yield: $72 \%, \mathrm{mp}: 147-149{ }^{\circ} \mathrm{C}$, IR $(\mathrm{KBr}): 3166\left(\mathrm{~N}-\mathrm{H}, \mathrm{NH}_{2}\right), 3078(\mathrm{C}-\mathrm{H}, \mathrm{Ar})$, $1688(\mathrm{C}=\mathrm{O}), 1636(\mathrm{C}=\mathrm{C}, \mathrm{Ar}), 1580(\mathrm{C}=\mathrm{N}), 1164(\mathrm{C}-\mathrm{O}) \mathrm{cm}^{-1} ;{ }^{1} \mathrm{H}$ NMR $\left(300 \mathrm{MHz}, \mathrm{DMSO}-\mathrm{d}_{6}\right)$ : $\delta$ ppm $9.71(\mathrm{~s}, 1 \mathrm{H}, \mathrm{NH}), 7.65(\mathrm{~s}, 1 \mathrm{H}, \mathrm{Ar}-\mathrm{H}), 7.62-7.44(\mathrm{~m}, 3 \mathrm{H}, \mathrm{Ar}-\mathrm{H}), 7.58(\mathrm{~d}, 2 \mathrm{H}, \mathrm{J}=7.2 \mathrm{~Hz}$, Ar-H), 7.40 (d, 2H, J = 7.2 Hz, Ar-H), $3.12\left(\mathrm{~s}, 3 \mathrm{H}, \mathrm{OCH}_{3}\right) \cdot{ }^{13} \mathrm{C}$ NMR (100 MHz, DMSO-d 6 ): $\delta$ ppm 169.1, 166.3, 162.4, 155.8, 154.3, 149.2, 146.4, 138.1, 129.2, 127.1, 125.2, 123.0, 120.8, 119.5, 118.1, 112.3, 46.4. MS: $393 \mathrm{~m} / \mathrm{z}\left(\mathrm{M}^{+}\right)$. Elemental analysis: calculated for $\mathrm{C}_{19} \mathrm{H}_{12} \mathrm{FN}_{5} \mathrm{O}_{2} \mathrm{~S}$ : C-58.01, H-3.07, F-4.83, N-17.80, O-8.13, S-5.15. Found: C-56.23, H-2.98, F-4.54, N-17.02, O7.84, S-5.02.

2-(3-Chloro phentlamino)-8-fluoro-[1,3,4] thiadiazolo-[3,2-a]pyrimidin-[5,4-c]quinolin-5-one (5e). Pale yellow solid, yield: $66 \%$, mp: $174-176{ }^{\circ} \mathrm{C}$, IR $(\mathrm{KBr}): 3184\left(\mathrm{~N}-\mathrm{H}, \mathrm{NH}_{2}\right), 3066(\mathrm{C}-\mathrm{H}$, Ar), $1669(\mathrm{C}=\mathrm{O}), 1640(\mathrm{C}=\mathrm{C}, \mathrm{Ar}), 1585(\mathrm{C}=\mathrm{N}) \mathrm{cm}^{-1} ;{ }^{1} \mathrm{H}$ NMR $\left(300 \mathrm{MHz}, \mathrm{DMSO}-\mathrm{d}_{6}\right): \delta \mathrm{ppm}$ 9.65 (s, 1H, NH), 7.71 (s, 1H, Ar-H), 7.69-7.44 (m, 6H, Ar-H), 7.58 (s, 1H, Ar-H). ${ }^{13} \mathrm{C}$ NMR $\left(100 \mathrm{MHz}, \mathrm{DMSO}-\mathrm{d}_{6}\right): \delta \mathrm{ppm} 167.0,163.0,162.4,158.6,150.2,144.7,142.0,136.7,129.4$, 126.3, 125.3, 123.4, 122.0, 121.7, 120.2, 116.3, 114.8, 113.1. MS: $397 \mathrm{~m} / \mathrm{z}\left(\mathrm{M}^{+}\right)$. Elemental analysis: calculated for $\mathrm{C}_{18} \mathrm{H}_{9} \mathrm{ClFN}_{5} \mathrm{OS}$ : C-54.35, H-2.28, Cl-8.91, F-4.78, N-17.60, O-4.02, S8.06. Found: C-52.69, H-2.20, Cl-8.12, F-4.68, N-16.98, O-3.87, S-7.85.

2-(4-Chloro phentlamino)-8-fluoro-[1,3,4] thiadiazolo-[3,2-a]pyrimidin-[5,4-c]quinolin-5-one (5f). Brown solid, yield: $68 \%$, mp: $123-125^{\circ} \mathrm{C}$, IR $(\mathrm{KBr}): 3192\left(\mathrm{~N}-\mathrm{H}, \mathrm{NH}_{2}\right), 3062(\mathrm{C}-\mathrm{H}, \mathrm{Ar})$, $1674(\mathrm{C}=\mathrm{O}), 1635(\mathrm{C}=\mathrm{C}, \mathrm{Ar}), 1592(\mathrm{C}=\mathrm{N}) \mathrm{cm}^{-1} ;{ }^{1} \mathrm{H}$ NMR $\left(300 \mathrm{MHz}, \mathrm{DMSO}-\mathrm{d}_{6}\right): \delta \mathrm{ppm} 9.66(\mathrm{~s}$, 1H, NH), 7.69 (s, 1H, Ar-H), 7.65-7.42 (m, 3H, Ar-H), 7.55 (d, 2H, J = 7.0 Hz, Ar-H), 7.48 (d, $2 \mathrm{H}, \mathrm{J}=7.0 \mathrm{~Hz}, \mathrm{Ar}-\mathrm{H}) .{ }^{13} \mathrm{C}$ NMR $\left(100 \mathrm{MHz}, \mathrm{DMSO}-\mathrm{d}_{6}\right): \delta \mathrm{ppm} 170.5,166.3,164.2,154.3$, 150.7, 145.3, 143.7, 134.6, 129.8, 126.3, 125.7, 121.8, 119.8, 117.4, 113.2, 112.0. MS: $397 \mathrm{~m} / \mathrm{z}$ $\left(\mathrm{M}^{+}\right)$. Elemental analysis: calculated for $\mathrm{C}_{18} \mathrm{H}_{9} \mathrm{ClFN}_{5} \mathrm{OS}$ : C-54.35, H-2.28, Cl-8.91, F-4.78, N17.60, O-4.02, S-8.06. Found: C-52.69, H-2.20, Cl-8.12, F-4.68, N-16.98, O-3.87, S-7.85.

2-(3-Nitro phentlamino)-8-fluoro-[1,3,4] thiadiazolo-[3,2-a]pyrimidin-[5,4-c]quinolin-5-one (5g). Greenish yellow solid, yield: 67\%, mp: 130-132 ${ }^{\circ} \mathrm{C}$, IR $(\mathrm{KBr}): 3168\left(\mathrm{~N}-\mathrm{H}, \mathrm{NH}_{2}\right), 3058(\mathrm{C}-$ $\mathrm{H}, \operatorname{Ar}), 1670(\mathrm{C}=\mathrm{O}), 1633(\mathrm{C}=\mathrm{C}, \operatorname{Ar}), 1625(\mathrm{~N}=\mathrm{O}), 1577(\mathrm{C}=\mathrm{N}) \mathrm{cm}^{-1} ;{ }^{1} \mathrm{H}$ NMR $(300 \mathrm{MHz}$, DMSO-d $\left._{6}\right): \delta$ ppm $9.78(\mathrm{~s}, 1 \mathrm{H}, \mathrm{NH}), 7.76(\mathrm{~s}, 1 \mathrm{H}, \mathrm{Ar}-\mathrm{H}), 7.71-7.54(\mathrm{~m}, 6 \mathrm{H}, \mathrm{Ar}-\mathrm{H}), 7.62(\mathrm{~s}, 1 \mathrm{H}$, 
Ar-H). ${ }^{13} \mathrm{C}$ NMR (100 MHz, DMSO-d $\left.\mathrm{d}_{6}\right): \delta$ ppm 167.2, 160.2, 159.8, 155.2, 151.4, 144.8, 141.0, 135.3, 129.8, 126.3, 125.7, 124.7, 122.3, 120.7, 119.7, 118.7, 115.6, 111.3. MS: $408 \mathrm{~m} / \mathrm{z}\left(\mathrm{M}^{+}\right)$. Elemental analysis: calculated for $\mathrm{C}_{18} \mathrm{H}_{9} \mathrm{FN}_{6} \mathrm{O}_{3} \mathrm{~S}: \mathrm{C}-52.94, \mathrm{H}-2.22, \mathrm{~F}-4.65, \mathrm{~N}-20.58, \mathrm{O}-11.75$, S7.85. Found: C-51.58, H-2.15, F-4.26, N-19.87, O-11.12, S-7.57.

2-(4-Nitro phentlamino)-8-fluoro-[1,3,4]thiadiazolo-[3,2-a]pyrimidin-[5,4-c]quinolin-5-one (5h). Brown solid, yield: 73\%, mp: $140-142{ }^{\circ} \mathrm{C}, \mathrm{IR}(\mathrm{KBr}): 3170\left(\mathrm{~N}-\mathrm{H}, \mathrm{NH}_{2}\right), 3054(\mathrm{C}-\mathrm{H}, \mathrm{Ar})$, $1668(\mathrm{C}=\mathrm{O}), 1630(\mathrm{C}=\mathrm{C}, \mathrm{Ar}), 1632(\mathrm{~N}=\mathrm{O}), 1587(\mathrm{C}=\mathrm{N}) \mathrm{cm}^{-1} ;{ }^{1} \mathrm{H}$ NMR $\left(300 \mathrm{MHz}, \mathrm{DMSO}-\mathrm{d}_{6}\right)$ : $\delta$ ppm $9.62(\mathrm{~s}, 1 \mathrm{H}, \mathrm{NH}), 7.66(\mathrm{~s}, 1 \mathrm{H}, \mathrm{Ar}-\mathrm{H}), 7.60-7.45(\mathrm{~m}, 3 \mathrm{H}, \mathrm{Ar}-\mathrm{H}), 7.51$ (d, 2H, J = $7.3 \mathrm{~Hz}$, Ar-H), 7.45 (d, 2H, J = 7.3 Hz, Ar-H). ${ }^{13} \mathrm{C}$ NMR (100 MHz, DMSO-d $\left.)_{6}\right): \delta$ ppm 165.6, 161.3, 157.4, 153.5, 150.2, 144.7, 143.4, 138.9, 125.7, 124.2, 121.4, 120.8, 119.8, 116.4, 114.9, 112.8 . MS: $408 \mathrm{~m} / \mathrm{z}\left(\mathrm{M}^{+}\right)$. Elemental analysis: calculated for $\mathrm{C}_{18} \mathrm{H}_{9} \mathrm{FN}_{6} \mathrm{O}_{3} \mathrm{~S}$ : C-52.94, H-2.22, F-4.65, N-20.58, O-11.75, S-7.85. Found: C-51.58, H-2.15, F-4.26, N-19.87, O-11.12, S-7.57.

\section{RESULTS AND DISCUSSION}

These initial reports stimulated us to integrate 1,3,4-thiodiazole and pyrimidine moieties into quinoline frame work, since these systems possess well documented biological activity. The target compounds, 2-arylamino-8-fluoro-[1,3,4]thiadiazolo-[3,2- $a]$ pyrimidin-[5,4-c]quinolin-5ones (5a-h) have been prepared by using commercially available 4-amino-8-fluoro-quinoline-3carboxylic acid ethyl ester (1) as raw material (Scheme 1). The initial intermediate, 8-fluoro-4methylsulfanylthiocarbonylamino-quinoline-3-carboxylic acid ethyl ester (2) has been prepared on constant stirring of a mixture of compound 1, carbon disulfide and dimethyl sulfoxide in aq. $\mathrm{NaOH}$ for $2 \mathrm{~h}$ at room temperature. Then compound $\mathbf{2}$ was reacted with hydrazine hydrate in ethanol solvent on uniform stirring under reflux for $6 \mathrm{~h}$ to get the next intermediate, 8-fluoro-4hydrazine thiocarbonylamino-quinoline-3-carboxylic acid ethyl ester (3). Further, the final intermediate, 3-amino-7-fluoro-2-mercapto-3H-pyrimido-[5,4-c]quinolin-4-one (4) for the synthesis of title compounds was prepared by the cyclization of compound $\mathbf{3}$ in ethanol solvent at reflux for $4 \mathrm{~h}$ with steady stirring. Finally, the compound $\mathbf{4}$ has been cyclized successively with a variety of aryl isothiocyanates and $\mathrm{K}_{2} \mathrm{CO}_{3}$ in dimethyl formamide under reflux for 20-22 $\mathrm{h}$ to get the title compounds, 2-arylamino-8-fluoro-[1,3,4]thiadiazolo-[3,2-a]pyrimidin-[5,4c] quinolin-5-ones (5a-h). The chemical structures of all newly discovered compounds were established by IR, ${ }^{1} \mathrm{H} \&{ }^{13} \mathrm{C}-\mathrm{NMR}$, mass spectral data and elemental analysis. Further, the target compounds were used to find their antimicrobial ability against different microorganisms.

Antibacterial activity

The in vitro antibacterial activity of all newly prepared compounds 2-arylamino-8-fluoro$[1,3,4]$ thiadiazolo-[3,2-a]pyrimidin-[5,4-c] quinolin-5-ones (5a-h) were evaluated against three gram-positive organisms like Bacillus subtilis, Micrococcus luteus, Staphylococcus aureus and five gram-negative bacteria such as Proteus vulgaris, Salmonella typhimurium, Pseudomonas aeruginosa, Escherichia coli and Salomonella paratyphi $A$ by broth dilution method [25] by using benzyl penicillin and streptomycin as standard drugs for comparison with title compounds. The results of the screening compounds are summarized in Table 1.

The in-vitro antibacterial activity of compounds $\mathbf{5 a - h}$ towards different tested bacterial organisms disclosed significant activity with a degree of variation (Table1). It is found that compound 5e displayed considerable activity against B. subtilis. Compounds $\mathbf{5 b}$ and $\mathbf{5 f}$ are significant active towards $M$. luteus compared to standard drug. Notable activity is also achieved for compound 5d against $S$. aureus. All the quinoline based 1,3,4-thiadiazolo pyrimidin derivatives (5a-h) have performed significant to moderate activity against gram-negative bacteria. Derivative $\mathbf{5 g}$ has displayed marked activity against $S$. typhimurium. Remaining 
derivatives such as, compounds $\mathbf{5} \mathbf{a}$ and $\mathbf{5 h}$ displayed least activity against all the tested microorganisms. Compounds $\mathbf{5 c}$ and $\mathbf{5 d}$ were showing significant activity against $E$. coli. The outstanding properties of this new class of antibacterial substances deserve further investigation in order to clarify the mode of action at molecular level, responsible for the activity observed.

Table 1. The in-vitro antibacterial activity of compounds 5a-h (zone of inhibition in $\mathrm{mm}$ ).

\begin{tabular}{|l|l|l|l|l|l|l|l|l|}
\hline Entry & B.s. & M.l. & S.a. & P.v. & S.t. & P.a. & E.c. & S.p. \\
\hline 5a & 10 & 14 & 13 & 12 & 14 & 13 & 15 & 17 \\
\hline $\mathbf{5 b}$ & 13 & 21 & 16 & 17 & 15 & 18 & 17 & 21 \\
\hline $\mathbf{5 c}$ & 18 & 19 & 18 & 16 & 18 & 17 & 19 & 17 \\
\hline $\mathbf{5 d}$ & 16 & 21 & 20 & 14 & 16 & 17 & 19 & 16 \\
\hline $\mathbf{5 e}$ & 25 & 19 & 15 & 13 & 15 & 15 & 21 & 21 \\
\hline $\mathbf{5 f}$ & 22 & 23 & 14 & 17 & 16 & 17 & 20 & 22 \\
\hline $\mathbf{5 g}$ & 15 & 15 & 15 & 18 & 24 & 18 & 18 & 19 \\
\hline $\mathbf{5 h}$ & 13 & 14 & 15 & 14 & 13 & 15 & 16 & 15 \\
\hline Benzyl pencillin & 24 & 26 & 28 & 27 & 28 & 29 & 28 & 29 \\
\hline Streptomycin & 29 & 28 & 31 & 30 & 31 & 28 & 32 & 28 \\
\hline
\end{tabular}

B.s. - B. subtilis; M.l. - M. luteus; S.a. - S. aureus; P.v. - P. vulgaris; S.t. - S. typhimurium; P.a. - P. aeruginosa; E.c. - E. coli; S.p. -S. paratyphi.

\section{CONCLUSIONS}

From the current investigation of anti-microbial and antibacterial screening of all the synthesized compounds, it was proved that all the compounds exhibiting better activity against positive organisms and five gram-negative bacteria. From these results, it was observed that $\mathbf{5 e}$ was exhibiting activity more than the slandered drug Benzyl Penicillin against $B$. subtilis and also considerable active against the second slandered drug Streptomycin. Similarly $\mathbf{5 b}$ and $\mathbf{5} \mathbf{f}$ are active against $M$. luteus, makeable activity of compound $\mathbf{5 d}$ against $S$. aureus. Compounds $\mathbf{5 c}$ and 5d were showing similar activity against E. coli. Also among $\mathbf{5 a - h}$, it was a common observation that 5a was showed lowest activity against all the bacteria. This was may be because of no substitution on phenyl ring. 5a-h exhibited the maximum activity by inhibiting the growth of all the bacteria to a greater extent in comparison with the standard drug Streptomycin. From the structure-activity relationship and to optimize the effectiveness of this series of molecules extensive study is also warranted to determine additional physicochemical and biological parameters.

\section{REFERENCES}

1. Mohammad, A.; Agarwal, R. Synthesis and anti-inflammatory activity of 5-(8-quinolinoxy methyl)-1,3,4-oxadiazole/1,3,4-thiadiazole and 1,2,4 (H) triazole. Ind. J. Heterocycl. Chem. 1998, 7, 225-228.

2. Abduallaa, M.A.; Ahmed, A.N.; El-zohry, M.F.; Omar, F. Coll. Czech. Chem. Commun. 1992, 57, 1547-1549.

3. Kuo, S.C.; Lee, H.Z.; Juang, J.P.; Lin, Y.T.; Wu, T.S.; Chang, J.J.; Lednicer, D.; Paull, K.D.; Lin, C.M.; Hamel, E.; Lee, K.H. Synthesis and cytotoxicity of 1,6,7,8-substituted 2(4'-substituted phenyl)-4-quinolones and related compounds: identification as antimitotic agents interacting with tubulin. J. Med. Chem. 1993, 36, 1146-1156.

4. Hamdi, N.; Lidrissi, C.; Saoud, M.; Nievas, A.R.; Zarrouk, H. Synthesis of some new biologically active coumarin derivatives. Chem. Heterocycl. Comp. 2006, 42, 320-325.

5. Gholap, A.R.; Toti, K.S.; Shirazi, F.; Kumari, R.; Bhat, M.K.; Deshpande, M.V.; Srinivasan, K.V. Synthesis and evaluation of antifungal properties of a series of the novel 2-amino-5- 
oxo-4-phenyl-5,6,7,8-tetrahydroquinoline-3-carbonitrile and its analogues. Bioorg. Med. Chem. 2007, 15, 6705-6715.

6. Elderfield, R.C.; Le Von, E.F. Synthesis of potential anticancer agents. 111. nitrogen mustards derived from 8-aminoquinolines. J. Org. Chem. 1960, 25, 1576-1583.

7. Garrouste, P.; Pawlowski, M.; Tonnaire, T.; Sicsic, S.; Dumy, P.; De Rosny, E.; ReboudRavaux, M.; Fulcrand, P.; Martinez, J. Synthesis and activity of HIV protease inhibitors. Eur. J. Med. Chem. 1998, 33, 423-436.

8. Narsinh, D.; Anamik, S. Synthesis and anti-HIV studies of some substituted pyrimidinediones, ethoxy pyrano[3,2-c]quinolines and hydrazino pyrano[3,2-c]quinolones. Ind. J. Pharm. Sci. 2001, 63, 211-215.

9. Pintilie, O.; Profire, L.; Sunel, V.; Popa, M.; Pui, A. Synthesis and antimicrobial activity of some new 1,3,4-thiadiazole and 1,2,4-triazole compounds having a D,L-ethionine moiety. Molecules 2007, 12, 103-113.

10. Mamolo, M.G.; Falagiani, V.; Zanpier, D.; Vio, L.; Banfi, F. Synthesis and antimycobacterial activity of [5-(pyridin-2-yl)-1,3,4-thiadiazol-2-ylthio]acetic acid arylidene-hydrazide derivatives. Farmaco 2001, 56, 587-592.

11. Zamani, K.; Faghifi, K.; Tefighi, I.; Sharlatzadeh, M.R. Synthesis and potential antimycotic activity of 4-substituted 3-(thiophene-2-yl-methyl)- $\Delta 2$-1,2,4-triazoline-5-thiones. Turk. $J$. Chem. 2004, 28, 95-101.

12. Zan, X.I.; Lai, L.H.; Jin, G.Y.; Zhong, Z.X. The presence of cyclosporine in body tissues and fluids during pregnancy. J. Agric. Food Chem. 2002, 50, 3757-3760.

13. Clerici, F.; Pocar, D.; Guido, M.; Loche, A.; Perlini, V.; Brufoni, M. Synthesis of 2-amino5-sulfanyl-1,3,4-thiadiazole derivatives and evaluation of their antidepressant and anxiolytic activity. J. Med. Chem. 2001, 44, 931-936.

14. Shenone, S.; Bruno, O.; Ranise, A.; Bondavalli, W.; Falcone, G.; Giordano, L.; Vitelli, M. 3Arylsulphonyl-5-arylamino-1,3,4-thiadiazol-2(3H)ones as anti-inflammatory and analgesic agents. Bioorg. Med. Chem. 2001, 9, 2149-2153.

15. Palaska, E.; Sahin, G.; Kelincen, P.; Durlu, N.T.; Altionax, G. Synthesis and antiinflammatory activity of 1-acylthiosemicarbazides, 1,3,4-oxadiazoles, 1,3,4-thiadiazoles and 1,2,4-triazole-3-thiones. II Farmaco 2002, 57, 101-107.

16. Desai, K.; Patel, R.; Chikhalia, K. Synthesis of pyrimidine based thiazolidinones and azitidinones: antimicrobial and antitubercular agents. J. Ind. Chem. 2006, 45(B), 773-778.

17. Amr, A.E.; Nermien, M.S.; Abdulla, M.M. Synthesis, reactions, and anti-inflammatory activity of heterocyclic systems fused to a thiophene moiety using citrazinic acid as synthon. Monatsh. Chem. 2007, 138, 699-707.

18. Fujiwara, N.; Nakajima, T.; Ueda, Y.; Fujita, H.; Kawakami, H. Novel piperidinyl pyrimidine derivatives as inhibitors of HIV-1 LTR activation. Bioorg. Med. Chem. 2008, 16, 9804-9816.

19. Ballell, L.; Field, R.A.; Chung, G.A.C.; Young, R.J. New thiopyrazolo[3,4-d]pyrimidine derivatives as anti-mycobacterial agents. Bioorg. Med. Chem. Lett. 2007, 17, 1736-1740.

20. Wagner, E.; Al-Kadasi, K.; Zimecki, M.; Sawka-Dobrowolska, W. Synthesis and pharmacological screening of derivatives of isoxazolo[4,5-d]pyrimidine. Eur. J. Med. Chem. 2008, 43, 2498-2504.

21. Cordeu, L.; Cubedo, E.; Bandres, E.; Rebollo, A.; Saenz, X.; Chozas, H.; Victoria Domínguez, M.; Echeverria, M.; Mendivil, B.; Sanmartin, C. Biological profile of new apoptotic agents based on 2,4-pyrido[2,3-d]pyrimidine derivatives. Bioorg. Med. Chem. 2007, 15, 1659-1669.

22. Gorlitzer, K.; Herbig, S.; Walter, R.D. Review on synthesis and various biological potential of thiazolopyrimidine derivatives. Pharmazie 1997, 52, 670-672.

23. Ukrainets, I.V.; Tugaibei, I.A.; Bereznykova, N.L.; Karvechenko, V.N.; Turov, A.V. Analgesic, anticonvulsant and anti-inflammatory activities of some synthesized 
benzodiazipine, triazolopyrimidine and bis-imide derivatives. Chem. Heterocycl. Comp. 2008, 5, 565-573.

24. Kurono, M.; Hayashi, M.; Miura, K.; Isogawa, Y.; Sawai, K. One pot synthesis of pyrimidine and bispyrimidine derivatives and their evaluation for anti-inflammatory and analgesic activities. Japan, Kokai Tokkyo Koho JP 1987, 62, 267-72; Chem. Abstr. 1988, $109,37832$.

25. National Committee for Clinical Laboratory Standards (NCCLS). Standard methods for dilution antimicrobial susceptibility tests for bacteria, which grows aerobically. Nat. Comm. Lab. Stands, Villanova 1982, 242-248. 